\title{
结合多注意力机制与时空图卷积网络的人体动作识别方法
}

\author{
李炫烨，郝兴伟，贾金公，周元峰 \\ (山东大学软件学院 济南 250101) \\ (hxw@sdu.edu.cn)
}

\begin{abstract}
摘 要: 人体动作识别因其难以结合时空域信息成为计算机视觉方向中一项具有挑战性的任务. 提出一个多注意力 时空图卷积网络, 其核心思想是根据时间序列信息和人体骨架的自然连接构建一个连通图, 然后利用具有多注意力 机制的时空图卷积网络自动地学习空间和时间特征并且优化该连通图, 最后实现对人体动作的预测. 引人图注意力 模块, 模型构建的图的拓扑结构在初始化后会随着网络训练的过程进行优化, 最终得到更适合表达人体动作的拓扑 结构. 此外, 加人通道注意力模块, 使网络能够更加注意相对重要的通道信息, 从而提取更有效描述动作的特征. 在 公认的大型数据集 NTU-RGBD 和 Kinetics 上进行了大量的实验, 结果表明该方法具有更高的识别准确率.
\end{abstract}

关键词：动作识别; 图卷积网络; 三维人体骨架

中图法分类号: TP391.41 DOI: 10.3724/SP.J.1089.2021.18640

\section{Human Action Recognition Method Based on Multi-Attention Mechanism and Spatiotemporal Graph Convolution Networks}

Li Xuanye, Hao Xingwei ${ }^{*}$, Jia Jingong, and Zhou Yuanfeng (School of Software, Shandong University, Jinan 250101)

\begin{abstract}
Human action recognition has become a challenging task in computer vision because it is difficult to combine spatiotemporal information. A multi-attention spatiotemporal graph convolution network is proposed. The core idea is to construct a connected graph according to the time series information and natural connection of human skeleton, and use the spatiotemporal graph convolution network with multi-attention mechanism to automatically learn spatial and temporal features and optimize the connected graph to realize prediction. Graph attention module is introduced, the topological structure of the graph constructed by the model will be optimized with the process of network training after initialization, then the topological structure which is more suitable for expressing human actions will be obtained. In addition, the channel attention module is added to make the network pay more attention to the important channel information, so as to extract the features of describing actions more effectively. A large number of experiments are carried out on the recognized large datasets: NTU-RGDB and Kinectics, which show that the method has higher recognition accuracy.
\end{abstract}

Key words: action recognition; graph convolution networks; 3D human skeleton

人体动作识别是计算机视觉中一个活跃的研 究领域, 因为它在视频监控、人机交互等领域具有
广泛的应用，所以越来越成为人们感兴趣的研究 方向. 动作识别方法可以根据所输人数据的形式

收稿日期: 2020-09-06; 修回日期: 2020-11-27. 李炫烨(1996-), 女, 硕士研究生, CCF 学生会员, 主要研究方向为人体动作识别; 郝兴伟(1966一), 男, 博士, 教授, 硕士生导师, CCF 会员, 论文通讯作者, 主要研究方向为 Web 挖掘; 贾金公(1995一), 男, 硕士研究 生, 主要研究方向为人体动作识别; 周元峰(1980-), 男, 博士, 教授, 博士生导师, CCF 高级会员, 主要研究方向为计算几何、图像 处理及医学影像处理. 
大体分为 2 类: 基于图像的方法 ${ }^{[-4]}$ 和基于骨架的 方法 ${ }^{[5-9]}$. 基于图像的方法一般使用 RGB 数据作为 输人, 通过提取图像特征进行动作识别. 基于骨架 的方法使用人体骨架数据(骨架数据由人体关节点 的二维或三维坐标组成)作为输人, 通过特征提取 对动作进行分类. 具有成本低、易获得等优点的深 度摄像头的出现和流行, 使获得人体骨架关节点 的精确坐标变得更加容易. 由于骨架数据具有鲁 棒性和对光照条件变化不敏感等特点, 在给定精 确的关节点坐标的情况下, 基于骨架数据的动作 识别方法的表现会更加出色和优异. 本文主要研 究的是基于三维人体骨架的动作识别技术.

传统的基于骨架的动作识别方法一般通过使 用人工设计的一系列特征表示模拟人的动作, 然 而这些方法具有一些缺点, 如有限的泛化能力和 没有良好地利用人体的空间关系信息等. 最近, 基 于深度学习的方法在动作识别领域中取得了很大 的进展. 例如, 文献[10]将骨架关节点的坐标通过 特定的转换形成张量并送人所设计的神经网络中 学习动作特征. 但是, 这类方法并未充分地利用人 体骨架的空间结构信息和时间动态信息. 近年来, 许多研究人员根据人体骨架序列数据的特性把数 据建模与图结构进行充分的融合, 提出了使用图 卷积网络 (graph convolutional networks, GCN $)^{[11]}$ 进 行动作预测的想法. 具体来说, 骨架数据不再被设 计成某种形式的张量作为网络的输人, 而是根据 人体的空间结构和序列的时间顺序形成能够描述 骨架序列的拓扑图, 然后在 GCN 中转换成能够表 示动作的特征向量, 进一步进行动作识别. 由于 $\mathrm{GCN}$ 能够充分利用人体的空间关系，因此该类方 法的性能有很大的提升. 然而, 固定的拓扑结构对 于描述多样性的动作样本并不是一个最好的选择, 它限制了节点间消息传递的范围. 例如, “踢”动作, 固定的图结构中没有 2 条腿部之间的物理连接, 它 无法关注整个骨架中更重要的腿部消息的传递. 因 此, 一个能够根据数据样本动态调整的图结构更适 合对多样性的动作进行建模. 另外, 之前的 GCN 忽 视了对不同通道的重视程度. 往往有的通道产生的 特征对于动作识别是非常重要的, 而有的通道中的 特征却只有微小的作用, 特征提取的过程应该更关 注那些重要的通道特征, 忽略不重要的通道信息.

综合以上问题，为了能够根据数据样本动态 地调整图结构, 本文提出了一种图注意力模块, 能 够在时空域中动态地、数据驱动地修改图的拓扑结 构, 从而可以更好地描述多样性的动作样本. 此
外, 考虑到在特征提取过程中产生的圥余特征会 影响最终的分类精度, 本文使用通道注意力模块, 可以对含有不同语义的通道使用注意力机制, 使 网络模型关注更重要的特征信息, 帮助提取与动 作更相关的特征, 消除冗余特征的影响.

本文方法首先将骨架数据送人网络. 在空间 图卷积层, 加人图注意力模块. 它由 2 部分组成, 第 1 部分是数据驱动图矩阵, 它根据骨架序列中的 人体物理结构和时间信息进行初始化, 然后在神 经网络训练的过程中动态地优化该图结构, 与固 定的图相比, 能够充分地传递没有直接相连的点 之间的消息, 并且动态地调整边的权重以获得更 好的动作表达能力. 第 2 部分是图注意力矩阵, 它 通过计算任意 2 个关节点间的相似性来形成, 共同 调整图结构. 另外, 网络使用了通道注意力模块, 它能够学习特征图中哪些通道的特征更需要被关 注, 该模块进一步提高了模型的性能, 使动作识别 的准确率有所增强. 然后, 进人时间图卷积层提取 时间特征. 最终, 经过分类器对动作进行分类并预 测. 为了评估该方法的有效性, 在 2 个公认的数据 集 NTU-RGBD ${ }^{[12]}$ 和 Kinetics ${ }^{[13]}$ 上进行了大量的实 验, 结果证明该方法优于最新的单流方法.

\section{1 相关工作}

由于骨架数据可以很容易地从高精度的深度 摄像设备和姿态估计算法 ${ }^{[14]}$ 中获得, 所以在动作 识别任务中有很多基于骨架的动作识别方法. 这 些方法大体可以分为传统的基于手工设计特征的 方法 ${ }^{[15-17]}$ 和基于深度学习的方法 ${ }^{[18-19]} 2$ 类.

传统的基于骨架的动作识别方法通常需要设 计手工特征对人体进行建模, 描述关节运动的动 态性. 例如, 文献[15]使用关节轨迹的协方差矩阵; 文献[16]使用关节点的相对位置对动作进行建模; 文献[17]使用身体各部分之间的旋转和平移等. 然 而, 这些方法的性能并不令人满意, 因为它们无法 同时考虑多种描述元素.

随着深度学习的发展, 基于深度学习的动作 识别方法也逐渐成为大家主要关注的研究方向. 基于骨架的动作识别任务在利用深度学习方法进 行建模时主要使用 2 种广泛流行的模型以端到端 的方式进行学习, 即卷积神经网络 (convolutional neural networks, CNN) 和递归神经网络(recurrent neural networks, RNN). 其中典型的使用 $\mathrm{CNN}$ 的方 法 ${ }^{[20-22]}$ 的主要思想是将骨架数据通过人工设计的 
变换规则转换为类似图像的特征图作为输人，然 后使用 $\mathrm{CNN}$ 进行特征提取, 达到动作识别的目的. 例如，文献[9]将每帧中任意 2 个连接的关节点间 的距离映射到图像上, 并将图像输人以 ImageNet 为基础的 $\mathrm{CNN}$ 模型中，以实现动作分类的目的. 文献[21]根据注意力机制设计了一种新颖的骨架 变换器模块，通过线性变换实现了对重要骨架关 节的自动重排和选择, 并利用 $\mathrm{CNN}$ 模型实现对动 作的分类. 该类方法主要是利用 $\mathrm{CNN}$ 模型在处理 图像识别问题上的优势，实现对骨架序列的动作 分类. 基于 RNN 的方法 ${ }^{[19,23-24]}$ 中，文献[23]的分层 RNN 将不同身体部位的特征进行分层组合. 在浅 层中，每个子网络在单个关节上提取特征，然后在 深层中融合这些特征表示. 当所有的节点信息被 融合后，网络进行最终的动作预测. 文献[19]在具 有长短期记忆的 RNN 基础上建立模型，该网络通 过使用注意力机制建立了 2 个子网络，分别对不同 帧和关节点赋予不同程度的关注，最终实现端到 端的行为识别. 这类方法利用的是 RNN 在处理时 序问题上的优势对骨架序列进行处理. 2 类方法虽 然都有广泛的应用，但是 CNN 和 RNN 都不能很好 地保留骨架的空间结构信息，因为以向量或类似 图像的二维网格形式描述骨架序列无法准确地表 示骨架数据的拓扑结构.

近年来, GCN 快速发展并应用于处理图数据. 它通常有 2 种构造思想, 分别遵循不同的原理. 一 种是谱域思想, 其原理是借助傅里叶变换, 类似地 在频域中执行图卷积; 另一种是空域思想，其原理 是直接对拓扑图的节点及其邻域使用卷积滤波器 来提取特征. 空域思想通常依靠手工设计的规则 提取和获得邻居节点. GCN 提出后, 文献[25]根据 注意力机制, 设计了图注意力网络, 通过叠加隐藏 的 self-attention 层, 将不同权重分配给邻域内的不 同节点, 最终在图结构数据上实现节点的分类. 文 献[26]首次提出一种基于 $\mathrm{GCN}$ 用于人体动作识别, 该模型称为时空图卷积网络 (spatial-temporal graph convolutional networks, ST-GCN). 随后，文献[27] 在此基础上提出了一个动作-结构 $\mathrm{GCN}$ 进行人体 动作识别和预测, 该模型利用注意力思想设计了 A-link 评估任意节点间的联系重要性程度, 并且 利用 S-link 获得图的更高阶的依赖关系，通过 2 种链接的组合学习动作的时空特征. 基于 $\mathrm{GCN}$ 的 方法利用图结构对骨架进行建模，通过在图上进 行卷积操作来提取特征, 进而完成对动作的识别 和预测

\section{2 基于多注意力机制的 ST-GCN}

\subsection{ST-GCN}

本节首先介绍原始的 ST-GCN 如何构建图，然 后描述在空间和时间维度上如何实现图卷积操作.

原始的骨架序列是由一组坐标数据组成的，它 可以用每帧中所有人体关节点的二维或三维坐标表 示. 考虑人体骨架本身就是一个拓扑结构，因此用 时空图对骨架序列中的关节点在时间和空间维度上 分别进行建模. 具体来说, 对于一个含有 $N$ 个节点 和 $T$ 帧的骨架序列，在该骨架序列上构建一个无向 图 $G=(V, E)$, 其中, $V=\left\{v_{t i} \mid t=1,2, \cdots, T, i=1,2, \cdots, N\right\}$ 代表节点集合，它包含了这个序列中所有的节点. 对于节点 $v_{t i}$ 上的属性, 它是由该点的空间坐标 $(x, y, z)$ 组成的特征向量. 边集合 $E$ 由 $E_{s}$ 和 $E_{t} 2$ 个部分组成， $E_{s}$ 表示在相同帧上的人体骨架关节 的自然连接, 它是帧内连接; $E_{t}$ 表示同一个关节点 在相邻帧上的连接, 它属于帧间连接. 图 1 所示为 一个构建好的时空图, 其中蓝色的点代表人体骨架 关节点, 蓝色的线代表人体的自然物理连接, 绿色 的线代表同一个关节点在相邻帧上的时间连接.

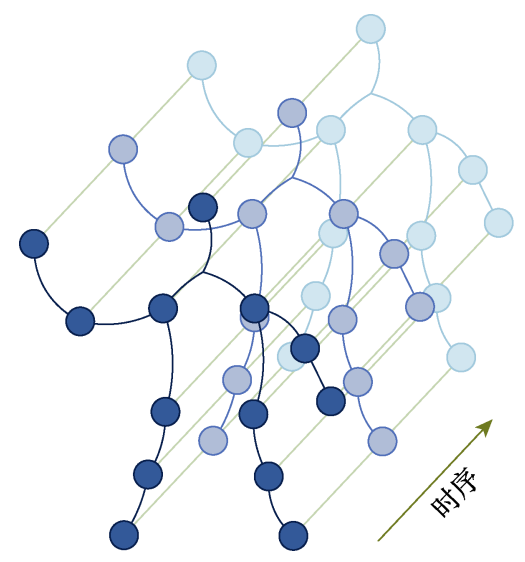

图 1 时空图

根据已定义好的图 $G$, 在空间维度上, 图卷 积运算有如下定义. 对于第 $\tau$ 帧上的顶点 $v_{\tau i}$, 它 可以表示为

$$
f_{\text {out }}\left(v_{\tau i}\right)=\sum_{v_{\tau j} \in S_{i}} \frac{1}{T_{i j}} f_{\text {in }}\left(v_{\tau j}\right) w\left(l_{i}\left(v_{\tau j}\right)\right)
$$

其中， $v$ 表示时空图上的顶点； $f_{\text {in }}$ 代表特征映射; $S_{i}$ 是目标顶点 $v_{\tau i}$ 卷积的采样区域，在这里为点 $v_{\tau i}$ 的一阶邻居节点 $v_{\tau j}$ 的集合; 权重函数 $w$ 用来提供 权重向量. 由于每个节点的邻居数目是不同的, 所 以 $S_{i}$ 中的数目是变化的, 而 $w$ 中权重向量的个数 
是不变的, 因此 $\mathrm{ST}-\mathrm{GCN}^{[26]}$ 使用映射函数 $l$ 为特征 向量分配权重. 具体来说, 将 $S_{i}$ 划分为 $k$ 个子集, 其中每个子集有自己的标签, 这样即可得到映射 $l_{i}$, 它将每个邻居节点映射到 $S_{i}$ 的某个子集当中. 如图 2 所示, $O$ 表示骨架的重心, 红色区域内是 $S_{i}$, 在这里 $k$ 设置为 3 , 因此 $S_{i}$ 由 3 个子集组成: $S_{i 1}$ 是目标节点本身(图 2 红色圆圈); $S_{i 2}$ 是向心节 点集(黄色圆圈), 它表示与目标节点相比这些邻居 节点距离重心 $(O)$ 的路径更短; $S_{i 3}$ 是离心节点集 (蓝色圆圈), 它表示与目标节点相比这些邻居节点 距离重心 $(O)$ 更远. $T_{i j}$ 表示顶点 $v_{\tau j}$ 所在 $S_{i}$ 子集的 基数. 对式(1)进行转换, 得到图卷积在空间维度 上实现的公式为

$$
f_{\text {out }}=\sum_{k}^{K_{v}} w_{k}\left(f_{\text {in }}\left(\widetilde{A_{k}} \odot M_{k}\right)\right)
$$

其中， $K_{v}$ 表示卷积核的大小; $\widetilde{\boldsymbol{A}}$ 是邻接矩阵 $\boldsymbol{A}$ 的 归一化形式; $\boldsymbol{M}$ 是一个可学习的权重矩阵; 符号 $\odot$ 表示点积. 使用式(2)就可以在空间维度实现图 卷积操作.

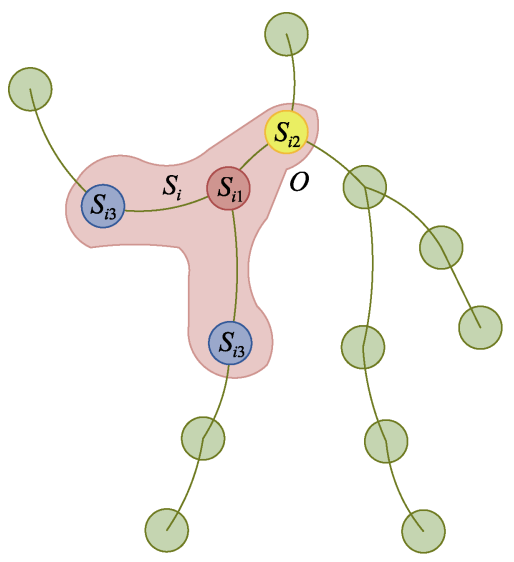

图 2 人体骨架空间域卷积示意图

图卷积在时间维度上的实现与经典的二维卷 积操作是类似的, 因为对于每个顶点 $v_{t i}$, 它在相 邻 2 个连续帧上对应的关节点是固定的, 即它在时 间轴上有 2 个邻居节点. 因此，在经过空间维度的 图卷积后, 对输出的特征图在时间维度进行普通 的二维卷积即可提取时间特征, 实现时间图卷积 运算.

\section{2 多注意力机制}

本节主要介绍基于原始 ST-GCN 引人的多注 意力机制, 其中分别介绍所提出的图注意力模块 和通道注意力模块.

\subsection{1 图注意力模块}

对空间图卷积层进行修改，引人图注意力模 块, 使模型不仅可以学习网络的参数, 还可以优化 连通图, 以得到更适合描述动作的图结构, 从而能 够更好地进行动作预测. 具体来说, 加人图注意力 模块后，空间图卷积公式可表示为

$$
f_{\text {out }}=\sum_{k}^{K_{v}} w_{k}\left(f_{\text {in }}\left(A_{k}^{\prime}+B_{k}\right)\right)
$$

与式(2)对比可以看出，图注意力模块包括 2 部分, 其中 $A^{\prime}$ 是一个数据驱动图矩阵, 它首先通 过第 2.1 节构建的图进行参数的初始化, 然后在网 络传播的过程中进行参数的更新. 与式(2)不同的 是, 式(2)中 $\widetilde{A}$ 是一个数值固定的邻接矩阵, $M$ 学 习的是连接的强度. 整个训练过程中无法产生新 的连接结构. 而使用新的矩阵 $\boldsymbol{A}^{\prime}$, 能够完全代替 $\widetilde{\boldsymbol{A}}$ 和 $\boldsymbol{M}$ 共同作用的效果. 矩阵 $\boldsymbol{A}^{\prime}$ 不仅能够充分地 利用初始的物理连接关系, 还能在训练过程中优 化连通图的拓扑结构, 更新边的权重, 从而实现了 用一个矩阵代替 2 个矩阵的效果. 并且, 由于 $A^{\prime}$ 是 完全根据训练数据所学习的, 所以该模型可以适 应各种不同的动作样本. 另外， $\boldsymbol{A}^{\prime}$ 在不同的卷积 层中是唯一的, 所以它在每层都是个性化的并且 含有不同语义的.

该模块的第 2 部分是图注意力矩阵 $\boldsymbol{B}$ ，它能 帮助模型更好地针对每个样本进行动作建模, 增 加模型的个性化. 具体地说, 对于某一输人特征 $f\left(v_{t i}\right)$ (节点 $v_{t i}$ 的特征), 首先分别使用 2 个卷积层 将 $f\left(v_{t i}\right)$ 映射为向量 $\boldsymbol{K}$ 和 $\boldsymbol{Q}$, 即

$$
\left\{\begin{array}{l}
\boldsymbol{K}_{t i}=\boldsymbol{W}_{K} f\left(v_{t i}\right) \\
\boldsymbol{Q}_{t i}=\boldsymbol{W}_{Q} f\left(v_{t i}\right)
\end{array} .\right.
$$

其中, $W_{K}$ 和 $W_{Q}$ 分别是 2 个卷积层对应的权重矩 阵. 接下来计算 $\boldsymbol{Q}_{t i}$ (节点 $v_{t i}$ 的 $\boldsymbol{Q}$ 向量) 和 $\boldsymbol{K}_{t j}$ (节点 $v_{t j}$ 的 $\boldsymbol{K}$ 向量) 的内积 $u_{(t, i) \rightarrow(t, j)}=\left\langle\boldsymbol{Q}_{t i}, \boldsymbol{K}_{t j}\right\rangle$. 其中, 节点 $v_{t i}$ 和 $v_{t j}$ 在同一个时间步内; 〈,〉代表内积符 号. 内积 $u_{(t, i) \rightarrow(t, j)}$ 称为节点 $v_{t i}$ 和节点 $v_{t j}$ 的相似性. 然后, 为了将 $u$ 的范围限制在 $0 \sim 1$, 使用 Softmax 函数对其进行归一化, 即

$$
\alpha_{(t, i) \rightarrow(t, j)}=\frac{\exp \left(u_{(t, i) \rightarrow(t, j)}\right)}{\sum_{n=1}^{N} \exp \left(u_{(t, i) \rightarrow(t, n)}\right)} .
$$

$\alpha$ 为内积 $u$ 归一化后的相似度, 即式(3)中矩阵 $\boldsymbol{B}$ 的元素. 由此可以看出, 矩阵 $\boldsymbol{B}$ 也是完全通过不 
同的动作样本学习得到的, 它能够有效地学习不 同动作中任意 2 个身体关节点的权重, 这种数据驱 动的方式增加了模型的灵活性和通用性，使模型 面对多样化的数据也能有效地进行动作预测.

添加上述图注意力模块, 使网络能在训练过 程中不断优化图结构, 适应多种样本的变化, 形成 最适合描述动作的拓扑结构, 最终提升模型的性 能, 使动作预测的结果更加准确.

\subsection{2 通道注意力模块}

在空间图卷积层中，经过图注意力模块后，得 到了初步提取的空间特征. 为了能够获取更好的 动作特征表示, 引人了通道域的注意力机制, 在空 间图卷积后添加了通道注意力模块. 该模块的结 构如图 3 所示. 首先, 对 $f_{\text {out }}$ 进行挤压操作 $\boldsymbol{F}_{1}$ 得到 矩阵 $\boldsymbol{Z}$. 然后经过 $\boldsymbol{F}_{2}$ 操作进人全连接层得到输出 矩阵 $\boldsymbol{S}, \boldsymbol{W}_{1}$ 与 $\boldsymbol{W}_{2}$ 分别为 2 个权重矩阵. 最终 $\boldsymbol{S}$ 与 原始输人相乘并通过残差方式相加得到最后输出 $f$. 经过实验证明，该模块有效地增强了模型特 征提取的能力, 进一步提高了动作预测的结果.

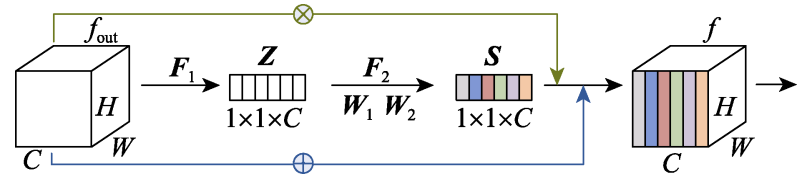

图 3 通道注意力模块

在卷积层中，往往每个层会产生新的通道，每 个通道对于关键信息的贡献有多有少，因此可以 为每个通道上的信号都添加一个权重, 代表该通 道与关键信息的相关度. 权重越大，代表该通道的 相关度越高, 需要更加注意该通道的信号; 相反, 则代表该通道的相关度越低，可相应地减少对该 通道的关注. 将第 2.2 .1 节的输出 $f_{\text {out }} \in \mathbb{R}^{H \times W \times C}$ 作 为该模块的输人进行挤压操作，实现全局信息嵌 人. 该操作可以表示为

$$
z_{c}=\frac{1}{H \times W} \sum_{i=1}^{H} \sum_{i=1}^{W} m_{c}(i, j) .
$$

其中, $m_{c} \in \mathbb{R}^{H \times W}$ 为经过该步骤输出的矩阵 $\boldsymbol{Z}$ 的 元素。该公式表示在时间和空间维度上进行平均 池化操作．接下来对输出 $\boldsymbol{Z}$ 进行变换，即

$$
\boldsymbol{S}=\sigma\left(\boldsymbol{W}_{2} \delta\left(\boldsymbol{W}_{1} \mathbf{Z}\right)\right) .
$$

其中, $\boldsymbol{W}_{1} \in \mathbb{R}^{\frac{C}{r} \times C}, \boldsymbol{W}_{2} \in \mathbb{R}^{\mathrm{C} \times \frac{C}{r}}$ 分别为全连接层的 2 个权重矩阵； $\sigma$ 代表 Sigmoid 激活函数; $\delta$ 代表 PReLU 激活函数. 也就是说, $\boldsymbol{Z}$ 首先经过一个全连
接层和一个 PReLU 激活函数, 再经过第 2 个全连 接层和一个 Sigmoid 激活函数, 然后得到输出 $\boldsymbol{S} \in \mathbb{R}^{1 \times 1 \times C}$. 最后, 将 $\boldsymbol{S}$ 与输人的特征图 $f_{\text {out }}$ 相乘, 并且通过残差的方式与原始的输人特征图相加, 得到该通道注意力模块的最后输出. 最终, 不同通 道的信号乘以不同的权重，从而可以增强网络对 关键通道的注意力, 以便更好地提取样本的时空 特征.

\section{3 多注意力 ST-GCN 的基本单元}

多注意力 ST-GCN 的每个基本单元都由 2 部分 构成, 分别是一个空间图卷积模块和一个时间图 卷积模块. 空间图卷积模块包含一个用来提取空 间特征的空间图卷积层、一个批标准化层和一个 ReLU 激活函数层. 其中, 图注意力模块位于空间 图卷积层, 该模块根据动作样本计算出图注意力 矩阵, 并与数据驱动图矩阵相加共同参与卷积运 算, 在该过程中不断更新参数, 帮助空间图卷积层 更好地提取骨架序列的空间特征。在空间图卷积 模块后是通道注意力模块. 时间图卷积模块连接 在通道注意力模块后, 它与空间图卷积模块类似, 包含一个用来提取时间特征的时间图卷积层、一个 批标准化层和一个 ReLU 激活函数层. 时间图卷积 层与传统的图像上的二维卷积类似, 即在 $C \times T \times N$ 的特征图上, 使用大小为 $K_{t} \times 1$ 的卷积核 对特征图进行卷积操作, 从而在时间维度上提取 特征. 最后, 在每个基本单元中使用残差连接使训 练更加稳定. 该模型的一个基本单元如图 4 所示.

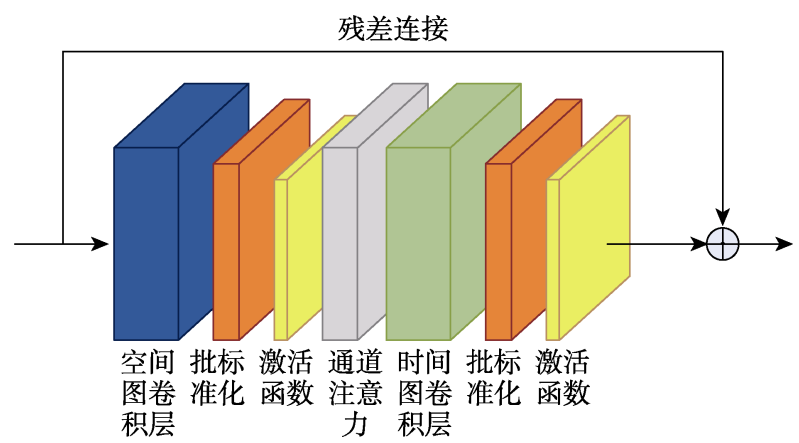

图 4 多注意力 ST-GCN 的基本单元

\section{4 多注意力 ST-GCN 的结构}

该网络由 9 个上述基本单元 $\left(B_{1} \sim B_{9}\right)$ 组成, 如 图 5 所示. 输人数据的通道数为 3 , 前 3 个基本单 元的输出通道数为 64 , 步长为 1 ; 中间 3 个基本单 元的输出通道数为 128 ; 最后 3 个单元的输出通道 数都是 256 , 并且每个基本单元都使用了残差机 制. 在 $B_{4}$ 和 $B_{7}$ 单元, 步长设置为 2 . 在将骨架数据 
送人基本单元之前, 首先对数据进行归一化操作, 使数据更加规范和便于处理, 这个操作在批标准 化层执行. 经过 9 个基本单元后, 将输出的特征图 送人到池化层进行全局平均池化来得到一个固定大 小的特征向量. 网络的最后是一个 Softmax 分类器, 它可以实现对动作的分类, 用来预测最终的结果.

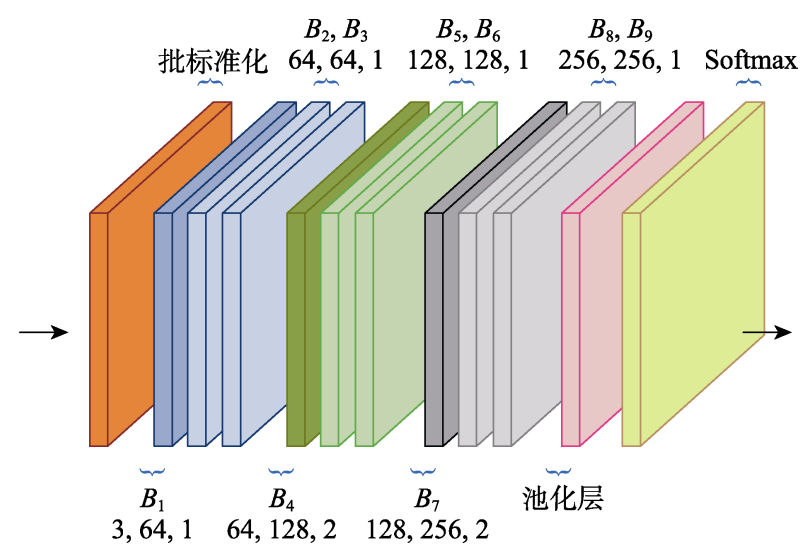

图 5 多注意力 ST-GCN 的结构

\section{3 实验结果与比较}

本文使用 2 个数据集 NTU-RGBD 和 Kinetics 进行消融实验, 以验证模型组成部分的有效性; 并 将其与最新的研究进行比较. 实验表明, 该模型在 2 个数据集上都取得了更高的识别准确率, 验证了 本文方法的有效性.

所有实验基于 Windows 10.0 系统，运行平台 为 NVIDIA GeForce GTX $1080 \mathrm{Ti}$ GPU. 采用 PyTorch 深度学习框架, Pycharm 作为集成开发环境.

\section{1 数据集}

NTU-RGBD 数据集是动作识别任务中应用最 广泛且非常具有挑战性的数据集. 该数据集的数 据由 3 台 Kinect V2.0 设备收集得来, 3 台摄像机在 同一高度但从不同水平角度 $\left(-45^{\circ}, 0^{\circ}, 45^{\circ}\right)$ 拍摄. NTU-RGBD 数据集总共包含 60 个不同的动作类 别, 共有 56880 个动作样本序列, 这些样本由 40 名 10 35 岁的志愿者在有限的实验室环境中拍摄 得来. 每个样本序列中有不超过 2 个动作实施对 象, 由所有帧的 25 个人体关节点的三维坐标组成, 动作种类既有单人的日常动作又有双人的日常动 作. 该数据集的作者提出了 2 个评价基准：(1) $\mathrm{CS}$ (cross subject)基准，该基准中的数据集分为训 练集和测试集 2 类，它们的动作执行主体不同; (2) $\mathrm{CV}$ (cross view)基准，该基准将在第 2 和第 3 设备
上收集到的数据用作训练集, 将在第 1 设备上收集 到的数据用作测试集.

Kinetics 是一个大规模的人体动作数据集, 它 包含 400 个动作类别共 300000 个视频片段, 这些 视频片段来源于 YouTube 视频, 并且种类繁多. 该 数据集只提供了没有骨架数据的原始视频序列. 文献[26]使用开源的 OpenPose 工具箱 ${ }^{[28]}$ 估计每个 视频帧上人体的 18 个关节点的位置. 使用作者发 布的数据集(Kinetics)评估本文的模型. 该数据集 共分为训练集(240000 个样本)和测试集(20000个 样本)2 部分, 按照文献[26]评估方法在训练集上训 练模型, 并展示了测试集中的 Top-1 和 Top-5 精度.

\section{2 消融实验}

本节以 NTU-RGBD 数据集上的 $\mathrm{CV}$ 基准为基 础, 检验了本文中模块的有效性. ST-GCN ${ }^{[26]}$ 在 NTU-RGBD 数据集上的原始性能为 $88.3 \%$, 采用 文献[29]中的数据预处理方法, 将其精度提高到 $92.7 \%$, 作为实验的基准.

\section{2 .1 数据驱动图矩阵}

本文在图注意力模块中设计了一个数据驱动 图矩阵 $\boldsymbol{A}^{\prime}$. 将它与原始的 ST-GCN ${ }^{[26]}$ 邻接矩阵进 行比较, 以显示其(ST-GCN+A)性能. 由表 1 可以 看出, 自适应学习图的拓扑结构有利于动作识别. 另外, 还测试了矩阵 $\boldsymbol{M}$ 在原始 $\mathrm{ST}-\mathrm{GCN}^{[26]}$ 中的重 要性, wo/M 代表在 ST-GCN ${ }^{[26]}$ 模型中删除矩阵 $\boldsymbol{M}$. 结果表明, 在给定每个连接的情况下, 权重参数的 调整有利于模型识别性能的提升.

\begin{tabular}{cc} 
表 $\mathbf{1}$ 数据驱动图矩阵的有效性 & $\%$ \\
\hline 方法 & $\mathrm{CV}$ \\
\hline ST-GCN ${ }^{[26]}$ & 92.7 \\
ST-GCN wo/M & 91.1 \\
ST-GCN $+\mathrm{A}$ & 93.6 \\
\hline
\end{tabular}

图 6 所示为数据驱动图矩阵的示例. 该图展示 了数据驱动图矩阵的第 2 个子集的初始化状态和 经过训练后的状态. 图 6a 所示的矩阵根据人体的 自然物理连接进行初始化, 图 $6 \mathrm{~b}$ 所示的矩阵是在 训练过程中动态调整后的邻接矩阵, 矩阵中每个 元素的灰度代表连接强度. 由此可以看出, 数据驱 动图矩阵经过训练后改变了原始邻接矩阵的连接, 更适合在动作识别任务中表示人体的拓扑结构.

\subsection{2 图注意力矩阵}

在第 2.2.1 节中, 不仅提出了数据驱动图矩阵, 并且图注意力矩阵 $\boldsymbol{B}$ 通过参与图卷积层的运算, 以获得更具动作表达能力的图结构表示. 将它与 


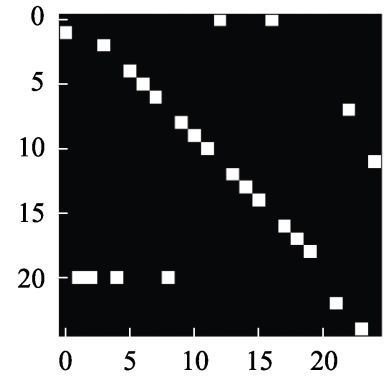

a. 初始化的数据驱动图矩阵

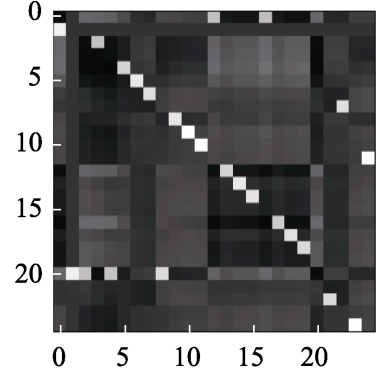

b. 经过训练的数据驱动图矩阵

图 6 数据驱动图矩阵的示例

原始的 ST-GCN ${ }^{[26]}$ 以及 ST-GCN $+\mathrm{A}$ 进行比较, 如表 2 所示. ST-GCN+B 代表在 ST-GCN 中添加图注意 力矩阵 $\boldsymbol{B} ; \mathrm{ST}-\mathrm{GCN}+\mathrm{A}+\mathrm{B}$ 代表在 $\mathrm{ST}-\mathrm{GCN}+\mathrm{A}$ 的模 型基础上再添加图注意力矩阵 $\boldsymbol{B}$, 即在 ST-GCN 中 添加完整的图注意力模块. 表 2 结果表明, 该图注 意力矩阵能够有效地帮助模型提高动作识别的准 确率, 获得更好的性能.

\begin{tabular}{ccc} 
表 2 & 图注意力矩阵的有效性 & $\%$ \\
\hline 方法 & $\mathrm{CV}$ \\
\hline ST-GCN ${ }^{[26]}$ & 92.7 \\
ST-GCN+B & 93.3 \\
ST-GCN + A & 93.6 \\
ST-GCN + A $+B$ & 94.1 \\
\hline
\end{tabular}

图 7 所示为图注意力矩阵的示例. 该图展示了 不同动作样本下图注意力矩阵的状态. 图 7a 所示 为动作 “喝水”的图注意力矩阵, 图 7b 所示为动作 “鼓掌”的图注意力矩阵, 矩阵中每个元素的灰度 代表连接强度. 由此可以看出, 不同动作状态下图 注意力矩阵的结构和连接强度是不同的, 数据驱 动的图注意力矩阵能够表达不同动作样本, 因此 在动作识别任务中具有更好的表现.
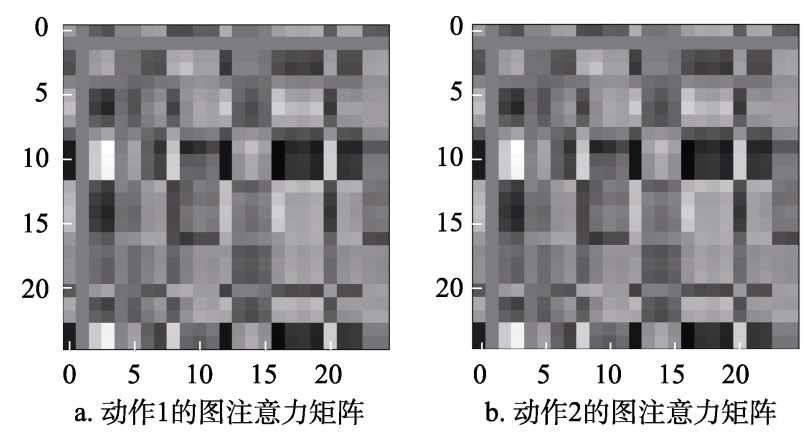

图 7 图注意力矩阵的示例

\subsection{3 通道注意力模块}

本节验证通道注意力模块的有效性, 结果如 表 3 所示. 首先测试基于 ST-GCN ${ }^{[26]}$ 的通道注意力 模块的贡献, 其结果显示为 $\mathrm{ST}-\mathrm{GCN}+\mathrm{C}$. 实验表

明, 该子模块有助于提高动作识别的准确率. 接下 来测试在模型 ST-GCN $+\mathrm{A}+\mathrm{B}$ 的基础上添加该子模 块的性能, 结果显示为 $\mathrm{ST}-\mathrm{GCN}+\mathrm{A}+\mathrm{B}+\mathrm{C}$ (本文方法 的完整模型), 即多注意力时空图卷积网络. 结果 表明, 该模块能够很好地嵌人本文的网络中, 帮助 提升网络的表现.

\begin{tabular}{|c|c|}
\hline 方法 & $\mathrm{CV}$ \\
\hline $\mathrm{ST}-\mathrm{GCN}^{[26]}$ & 92.7 \\
\hline $\mathrm{ST}-\mathrm{GCN}+\mathrm{C}$ & 93.6 \\
\hline $\mathrm{ST}-\mathrm{GCN}+\mathrm{A}+\mathrm{B}$ & 94.1 \\
\hline ST-GCN+A+B+C(本文) & 94.7 \\
\hline
\end{tabular}

\section{3 实现细节和实验结果比较}

所有实验的批大小(batchsize)设置为 64 , 优化 策略采用动量为 0.9 的随机梯度下降算法, 并选择 交叉熵作为损失函数反向传播梯度, 权重衰减设 置为 0.000 1. 对于 NTU-RGBD 数据集, 每个样本 中的最大帧数为 300 . 初始学习率设为 0.1 , 在第 30 次和第 40 次训练时下降 $10 \%$, 训练过程在第 50 次结束. 对于 Kinetics 数据集, 从输人的骨架序列 中随机选择 150 帧, 初始学习率也被设置为 0.1 , 在第 45 次和第 55 次训练时下降 $10 \%$, 训练在第 65 次结束.

在 NTU-RGBD 和 Kinetics 数据集上, 将最终 的模型与最新的基于骨架的动作识别方法进行比 较, 比较结果分别显示在表 4 和表 5 中. 其中, 用

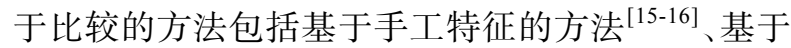
RNN 的方法 ${ }^{[6,12,17,23-24]}$ 、基于 $\mathrm{CNN}$ 的方法 ${ }^{[9,21-22,30]}$ 和基于 $\mathrm{GCN}$ 的方法 ${ }^{[22-27,31]}$. 本文模型在 2 个数据

表 4 在 NTU-RGBD 数据集上的准确率 \%

\begin{tabular}{lcc}
\hline \multicolumn{1}{c}{ 方法 } & CS & CV \\
\hline Lie Group $^{[15]}$ & 50.1 & 82.8 \\
HBRNN $^{[23]}$ & 59.1 & 64.0 \\
ST-LSTM $^{[17]}$ & 69.2 & 77.7 \\
VA-LSTM $^{[6]}$ & 79.2 & 87.7 \\
SRN+TSL $^{[24]}$ & 84.8 & 92.4 \\
TCN $^{[30]}$ & 74.3 & 83.1 \\
Clips+CNN+MTLN $^{[9]}$ & 79.6 & 84.8 \\
CNN+Motion+Trans $^{[21]}$ & 83.2 & 89.3 \\
3scale ResNet152 & 85.0 & 92.3 \\
ST-GCN & 81.5 & 88.3 \\
DPRL+GCNN & 83.5 & 89.8 \\
AS-GCN & 86.8 & 94.2 \\
本文 & 87.2 & 94.7 \\
\hline
\end{tabular}


表 5 在 Kinetics 数据集上的准确率 \%

\begin{tabular}{lcc}
\hline \multicolumn{1}{c}{ 方法 } & Top-1 & Top-5 \\
\hline Feature Enc $^{[16]}$ & 14.9 & 25.8 \\
Deep LSTM $^{[12]}$ & 16.4 & 35.3 \\
TCN $^{[30]}$ & 20.3 & 40.0 \\
ST-GCN $^{[26]}$ & 30.7 & 52.8 \\
AS-GCN & 34.8 & 56.5 \\
本文 & 34.7 & 57.8 \\
\hline
\end{tabular}

集上都达到了更高的准确率，具有更好的性能，这 有效地证明了本文模型的优越性.

\section{4 结 语}

本文提出了一种新的多注意力时空图卷积神 经网络，用于基于骨架的人体动作识别技术. 骨架 数据的拓扑结构被参数化并嵌人网络模型, 同时 使用图注意力机制加以调整，以便与其他网络参 数共同学习和更新. 这种数据驱动的方法提高了 模型的灵活性和泛化能力，同时也证明了自适应 学习的图结构比基于人体的固定图结构更适合动 作识别任务. 此外，在每个空间图卷积层中嵌人一 个通道注意力模块, 使模型更加关注重要的通道 特征，进一步提高了模型的性能. 最后，在 2 个具 有挑战的动作识别数据集 NTU-RGBD 和 Kinetics 上对模型进行了评估，实验证明它在 2 个数据集上 都获得了更高的识别精度. 未来的工作可以集中 在如何更好地融合和利用 RGB 数据和骨架数据上, 进一步改善和提高模型的性能.

\section{参考文献(References):}

[1] Guo G D, Lai A. A survey on still image based human action recognition[J]. Pattern Recognition, 2014, 47(10): 3343-3361

[2] Poppe R. A survey on vision-based human action recognition[J]. Image \& Vision Computing, 2010, 28(6): 976-990

[3] Zhu F, Shao L, Xie J, et al. From handcrafted to learned representations for human action recognition: a survey[J]. Image and Vision Computing, 2016, 55(2): 42-52

[4] Turaga P, Chellappa R, Subrahmanian V S, et al. Machine recognition of human activities: a survey[J]. IEEE Transactions on Circuits and Systems for Video Technology, 2008, 18(11): 1473-1488

[5] Graves A, Mohamed A, Hinton G. Speech recognition with deep recurrent neural networks[C] //Proceedings of the IEEE International Conference on Acoustics, Speech and Signal Processing. Los Alamitos: IEEE Computer Society Press, 2013: 6645-6649

[6] Zhang P F, Lan C L, Xing J L, et al. View adaptive neural net- works for high performance skeleton-based human action recognition[C] //Proceedings of the IEEE International Conference on Computer Vision. Los Alamitos: IEEE Computer Society Press, 2017: 2136-2145

[7] Sainath T N, Vinyals O, Senior A, et al. Convolutional, long short-term memory, fully connected deep neural networks[C] //Proceedings of the IEEE International Conference on Acoustics, Speech and Signal Processing. Los Alamitos: IEEE Computer Society Press, 2015: 4580-4584

[8] Li S, Li W Q, Cook C, et al. Independently recurrent neural network (IndRNN): building a longer and deeper RNN[C] //Proceedings of the IEEE Conference on Computer Vision and Pattern Recognition. Los Alamitos: IEEE Computer Society Press, 2018: 5457-5466

[9] Ke Q H, Bennamoun M, An S J, et al. A new representation of skeleton sequences for 3D action recognition[C] //Proceedings of the IEEE Conference on Computer Vision and Pattern Recognition. Los Alamitos: IEEE Computer Society Press, 2017: 4570-4579

[10] Li C K, Hou Y H, Wang P C, et al. Joint distance maps based action recognition with convolutional neural networks[J]. IEEE Signal Processing Letters, 2017, 24(5): 624-628

[11] Kipf $T$ N, Welling M. Semi-supervised classification with graph convolutional networks[OL]. [2020-09-06]. https://arxiv. org/pdf/1609.02907.pdf

[12] Shahroudy A, Liu J, Ng T T, et al. NTU RGB+D: a large scale dataset for 3D human activity analysis[C] //Proceedings of the 29th IEEE Conference on Computer Vision and Pattern Recognition. Los Alamitos: IEEE Computer Society Press, 2016: 1010-1019

[13] Kay W, Carreira J, Simonyan K, et al. The kinetics human action video dataset[OL]. [2020-09-06]. https://arxiv.org/pdf/ 1705.06950.pdf

[14] Fu L R, Zhang J G, Huang K Q. Beyond tree structure models: a new occlusion aware graphical model for human pose estimation[C] //Proceedings of the IEEE International Conference on Computer Vision. Los Alamitos: IEEE Computer Society Press, 2015: 1976-1984

[15] Vemulapalli R, Arrate F, Chellappa R. Human action recognition by representing $3 \mathrm{D}$ human skeletons as points in a lie group[C] //Proceedings of the IEEE Conference on Computer Vision and Pattern Recognition. Los Alamitos: IEEE Computer Society Press, 2014: 588-595

[16] Fernando B, Gavves E, José Oramas M, et al. Modeling video evolution for action recognition[C] //Proceedings of the IEEE Conference on Computer Vision and Pattern Recognition. Los Alamitos: IEEE Computer Society Press, 2015: 5378-5387

[17] Liu J, Shahroudy A, Xu D, et al. Spatio-temporal LSTM with trust gates for 3D human action recognition[C] //Proceedings of the 14th European Conference on Computer Vision. Heidelberg: Springer, 2016: 816-833

[18] Huang Z W, Wan C D, Probst T, et al. Deep learning on lie groups for skeleton-based action recognition[C] //Proceedings of the 30th IEEE Conference on Computer Vision and Pattern Recognition. Los Alamitos: IEEE Computer Society Press, 2017: 1243-1252

[19] Song S J, Lan C L, Xing J L, et al. An end-to-end spa- 
tio-temporal attention model for human action recognition from skeleton data[C] //Proceedings of the 31st AAAI Conference on Artificial Intelligence. San Francisco: AAAI Press, 2016: $4263-4270$

[20] Liu M Y, Liu H, Chen C. Enhanced skeleton visualization for view invariant human action recognition[J]. Pattern Recognition, 2017, 68: 346-362

[21] Li C, Zhong Q Y, Xie D, et al. Skeleton-based action recognition with convolutional neural networks[OL]. [2020-09-06]. https://arxiv.org/pdf/1704.07595.pdf

[22] Li B, He M Y, Cheng X L, et al. Skeleton based action recognition using translation-scale invariant image mapping and multi-scale deep CNN[OL]. [2020-09-06]. https://arxiv.org/pdf/ 1704.05645.pdf

[23] Du Y, Wang W, Wang L. Hierarchical recurrent neural network for skeleton based action recognition[C] //Proceedings of the IEEE Conference on Computer Vision and Pattern Recognition. Los Alamitos: IEEE Computer Society Press, 2015: 1110-1118

[24] Si C Y, Jing Y, Wang W, et al. Skeleton-based action recognition with spatial reasoning and temporal stack learning[C] //Proceedings of the 15th European Conference on Computer Vision. Heidelberg: Springer, 2018: 106-121

[25] Velikovi P, Cucurull G, Casanova A, et al. Graph attention networks[OL]. [2020-09-06]. https://arxiv.org/pdf/1710.10903.pdf

[26] Yan S J, Xiong Y J, Lin D H. Spatial temporal graph convolutional networks for skeleton-based action recognition[C]
//Proceedings of the 32nd AAAI Conference on Artificial Intelligence. San Francisco: AAAI Press, 2018: 7444-7452

[27] Li M S, Chen S H, Chen X, et al. Actional-structural graph convolutional networks for skeleton-based action recognition[C] //Proceedings of the IEEE Conference on Computer Vision and Pattern Recognition. Los Alamitos: IEEE Computer Society Press, 2019: 3595-3603

[28] Cao Z, Simon T, Wei S E, et al. Realtime multi-person 2D pose estimation using part affinity fields[C] //Proceedings of the IEEE Conference on Computer Vision and Pattern Recognition. Los Alamitos: IEEE Computer Society Press, 2017: 1302-1310

[29] Shi L, Zhang Y F, Cheng J, et al. Two-stream adaptive graph convolutional networks for skeleton-based action recognition[C] //Proceedings of the IEEE Conference on Computer Vision and Pattern Recognition. Los Alamitos: IEEE Computer Society Press, 2019: 12026-12035

[30] Kim T S, Reiter A. Interpretable 3D human action analysis with temporal convolutional networks[C] //Proceedings of the IEEE Conference on Computer Vision and Pattern Recognition Workshops. Los Alamitos: IEEE Computer Society Press, 2017: 1623-1631

[31] Tang Y S, Tian Y, Lu J W, et al. Deep progressive reinforcement learning for skeleton-based action recognition[C] //Proceedings of the IEEE Conference on Computer Vision and Pattern Recognition. Los Alamitos: IEEE Computer Society Press, 2018: 5323-5332 